\title{
Monitoring the synchrotron and Compton emission of PKS 2155-304 Fermi-LAT and RXTE long term observations
}

\author{
D. A. Sanchez ${ }^{1}$, B. Giebels ${ }^{2}$, D. Thompson ${ }^{4}$, G. Tosti $^{5}$, B. Lott ${ }^{6}$, on behalf of the
} Fermi-LAT collaboration

${ }^{1}$ Max-Planck-Institut für kernphysik, P.O. Box 103980, D 69029 Heidelberg, Germany

${ }^{2}$ Laboratoire Leprince-Ringuet, École Polytechnique, CNRS/IN2P3, France

${ }^{3}$ NASA Goddard Space Flight Center, Greenbelt, MD 20771

${ }^{4}$ Dipartimento di Fisica, Università degli Studi di Perugia, I-06123 Perugia, Italy

${ }^{5}$ CNRS/IN2P3, Centre dâĂŹÂt'Etudes Nucléaires Bordeaux Gradignan, France

E-mail: david.sanchez@mpi-hd.mpg.de

PKS 2155-304 is a well known GeV-TeV source and one of the brightest blazars in the Fermi sky. More than two years of Fermi operations provides the most extensive light curve of this object between $200 \mathrm{MeV}$ and $300 \mathrm{GeV}$. Together with monitoring from RXTE, these data allow the study of the temporal and spectral behavior of PKS 2155-304 in the two energy bands. A time dependent SSC model is used to fit the time-averaged SED (i) to reproduce the temporal behavior of a particular period, and (ii) to interpret the statistical properties of the light curves. Our analysis provides a complete picture of the emission mechanisms on long time scales for PKS 2155-304.

25th Texas Symposium on Relativistic Astrophysics - TEXAS 2010

December 06-10, 2010

Heidelberg, Germany 


\section{Introduction}

Blazars emit electromagnetic radiation over 20 decades of energy from radio to $\mathrm{TeV}$ energy for the most energetic ones. Their spectrum is dominated by non-thermal emission of relativistic electrons with a maximal energy $\gamma_{e}=E / m_{e} c^{2}$ of $10^{6}-10^{7}$. A subset of blazars, mainly BL Lac objects, has been detected at Very High Energy (VHE, $E>100 \mathrm{GeV}$ ) during the past 20 years ${ }^{1}$. The vast majority have a spectrum well described by a power law with an index greater than 2 in this energy range.

In a synchrotron self-Compton (SSC) scenario, the X-ray and $\mathrm{TeV}$ radiations are believed to be emitted by the same population of high energy electrons. Correlations between the two wavebands have been reported in several object, supporting the view of a co-spatial emission. The $\mathrm{GeV}$ photons are produced by lower energy electrons and the link between the $\mathrm{X}$-ray and the $\mathrm{GeV}$ is less known.

PKS 2155-304 ( $z=0.117)$ is a well studied BL Lac object. Classified as a high frequency peak BL Lac thanks to X-ray observations of HEAO-1 [11], PKS 2155-304 was detected in VHE in 1999 [4]. To probe possible correlations and have an insight onto the emission mechanism of PKS 2155304, the source was the target of monitoring observations with RXTE $(2-10 \mathrm{keV})$ and Fermi-LAT (200 MeV $-300 \mathrm{GeV}$ ) from February 2009 to January 2010. Preliminary results without the full RXTE data set has been reported in [10]. Here, we present the results of this campaign.

\section{Observation and analysis}

Fermi-LAT: The Large Area Telescope (LAT), one of the two instruments on board Fermi , is a pair converter telescope with its main characteristics describe in [2]. The LAT detects $\gamma$-rays from $\sim 20 \mathrm{MeV}$ to $300 \mathrm{GeV}$ and the full sky is observed every 3 hours.

For this study, data taken between MJD 54682 and MJD 55412 (August 4, 2008 - August 4, 2010) were used. Events from the DIFFUSE class with a reconstructed energy between $200 \mathrm{MeV}$ and $300 \mathrm{GeV}$ and coming from an Region Of Interest (ROI) of a radius of 10 degrees around the coordinates of PKS 2155-304 have been selected. To avoid earth albedo contamination, a cut on the zenith angle $\left(z<105^{\circ}\right)$ was made. The analysis was perform with the ScienceTools V9R17P0 and the IRFs P6_V3 to described the detector.

The sky model consists of a Galactic component, prepared with the GALPROP model [12] and an extragalactic component modeled with the isotropic spectral template isotropic_iem_v02.txt. All the point-like sources in the ROI, appearing in a internal version of the 2 years catalog, were included. Each spectra is described by a simple power law with the total flux above $200 \mathrm{MeV}$ and spectral index as free parameters. A unbinned maximum likelihood method, implemented in the gt like tool, was used to determine the parameters which best match the data.

RXTE : The RXTE observations consist of 82 pointed observations for a total of $2.5 \times 10^{5}$ seconds and took place between MJD 54897.5 to MJD 55196.5. Each observation lasts 4ks and occurred approximately every 3 days. The PCA data were extracted and analyzed using XSPEC [3], and the spectrum was corrected for Galactic absorption using a column density $N_{\mathrm{H}}=1.48 \times 10^{20} \mathrm{~cm}^{-2}$.

\footnotetext{
${ }^{1}$ Online catalogs such as TeVCat http: //tevcat.uchicago. edu presents updated view of the TeV sky.
} 


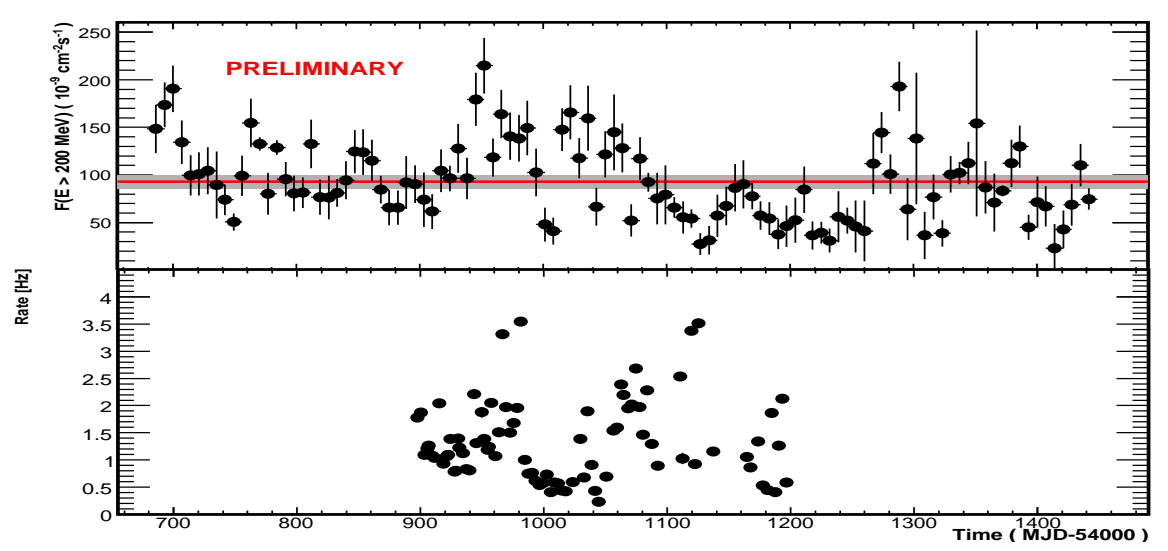

Figure 1: Top panel: Fermi Light curve with 7 days bins. The read line is the time average flux measured and the gray area give the corresponding error. Bottom panel: RXTE light curve between February 2009 and January 2019

\section{Temporal behaviors}

The Fermi light curve, with 7 days wide time bins, is shown in Figure 1 (top panel). In order to quantify the source variability, we use the normalized excess variance [13] defined by:

$$
\mathrm{F}_{\mathrm{var}}=\sqrt{\frac{S^{2}-\sigma_{e r r}^{2}}{\bar{f}^{2}}}
$$

where $f$ is the observed flux with measurement error $\sigma_{i}^{\text {err }} . S^{2}$ is the variance of $f$ and $\sigma_{e r r}^{2}$ is the average of $\sigma_{i}^{\text {err }}$. This quantity is suitable when one wants to compare data from different energy bands and different instruments. The source clearly shows variability with $F_{v a r}=0.35 \pm 0.03$ and order-of-magnitude flux variations.

To probe a possible energy dependent variability, the wide energy range of the Fermi-LAT has been split in two band $(0.2-1 \mathrm{GeV}$ and $1-300 \mathrm{GeV})$. The results for each are given in Table 1 and shown in Figure 2. On a timescale of 7 days, PKS 2155-304 exhibits more variability at low energy. This behavior is compatible with the value of $F_{\text {var }}$ measured by H.E.S.S, during the 2008 campaign, which is below the value measured in the $1-300 \mathrm{GeV}$ energy range. This is subject to caution due to the different timescales used in the different light curves to derive the variability.

In X-rays, the light curve was binned with 2 timescales. To match the Fermi timescale, we used a binning of 7 days and, to probe even smaller timescales, a 3 days wide binned light curve was computed. For each timescale, the source is variable with $F_{v a r}=0.47 \pm 0.01$ for the 7 days light curve. The value of $\mathrm{F}_{\mathrm{var}}$, using the two different binning time scales, and computed for three energy bins (2-4 keV, 4-6 keV and 6-10 keV), is given in Table 1 .

$\mathrm{F}_{\mathrm{var}}$ is found to be dependent of the energy $\mathrm{F}_{\mathrm{var}}(E) \propto E^{\kappa}$ with an index $\kappa=0.24 \pm 0.02(7$ days) and $\kappa=0.26 \pm 0.02$ (3 days) (Figure 2 ).. This behavior has been reported by several authors for different sources ([9] PKS 2155-304, [5] Markarian 421) with comparable values for $\kappa$. This fit is also compatible with the value of $F_{v a r}$ found in 2008 in the optical band [1] even if the data are not contemporaneous and not based on the same time binning. 


\begin{tabular}{|c||c|c|c|c|c|}
\hline Timescale & $2-4 \mathrm{keV}$ & $4-6 \mathrm{keV}$ & $6-10 \mathrm{keV}$ & $0.2-1 \mathrm{GeV}$ & $1-300 \mathrm{GeV}$ \\
\hline 3 days & $0.49 \pm 0.009$ & $0.548 \pm 0.005$ & $0.66 \pm 0.01$ & $\ldots$ & $\ldots$ \\
\hline 7 days & $0.395 \pm 0.009$ & $0.443 \pm 0.005$ & $0.548 \pm 0.005$ & $0.46 \pm 0.04$ & $0.38 \pm 0.03$ \\
\hline
\end{tabular}

Table 1: Measured values of $F_{v a r}$ for 2 timescales and for different energy bands. The statistic is not sufficient to compute this quantity with 3 days light curves above $0.2 \mathrm{GeV}$.
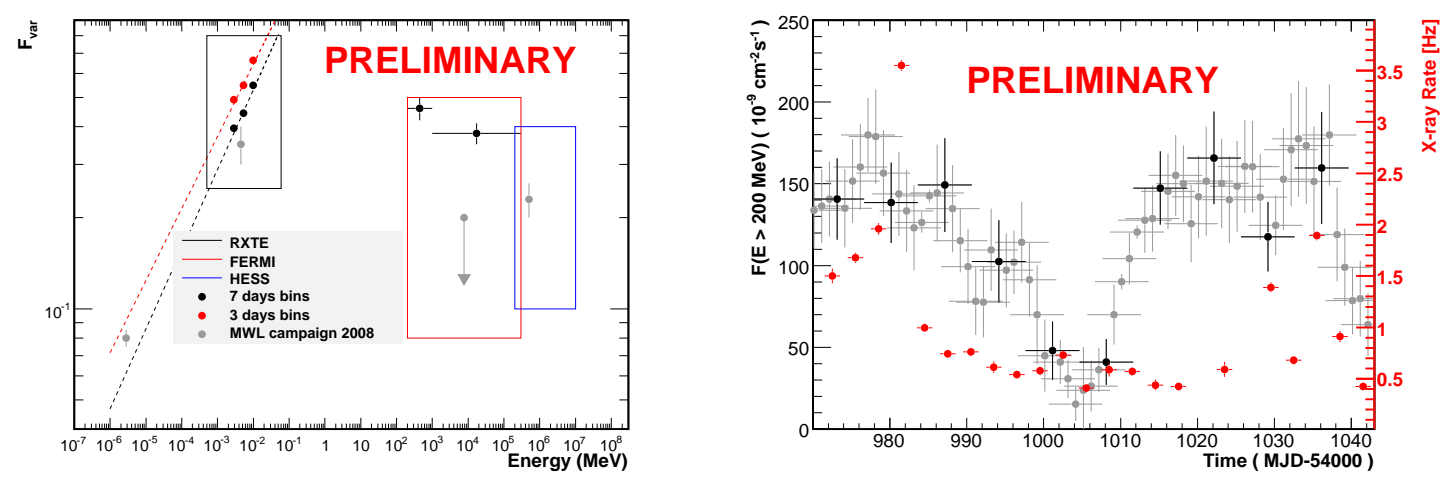

Figure 2: Left: $F_{\text {var }}$ as a function of the energy. Black and red points were obtained during this campaign. Right:Fermi (black/gray) and RXTE (red) light curves of the period MJD 54970-55040. The gray data points are the overlapping 7 day bins (see text).

During the period MJD 54970-55040, the X-ray and $\gamma$-ray fluxes reached both their highest and lowest states of the campaign, making this period very interesting. Figure 2 shows the X-ray (red) and $\mathrm{GeV}$ (Black/Grey) light curves superimposed. In this Figure, the Fermi-LAT light curve was obtained with overlapping 7 days bins with a delay of 1 day between 2 bins, allowing us to take advantage of all the available statistics [8].

On MJD 54981.5, the X-ray flux, at a level of $5.5 \times 10^{-11} \mathrm{erg} \mathrm{cm}^{-2} \mathrm{~s}^{-1}$, dropped by a factor 3 in 3 days and reached a plateau at 0.6 counts s${ }^{-1}$ (corresponding to $1.5 \times 10^{-12} \mathrm{erg} \mathrm{cm}^{-2} \mathrm{~s}^{-1}$ ) for 40 days before a small increase. During the same time, the $\mathrm{GeV}$ flux decreased by a factor 9 with a timescale of $15.0 \pm 2.2$ days and increased back to almost its previous value in $9.8 \pm 1.5$ days (Figure 2). The following sequence appears: the gamma-rays decay after the X-rays, but they ramp up again before the $\mathrm{X}$-rays.

\section{Spectral Energy Distribution}

The Fermi time average spectrum is compatible at a level of $3.1 \sigma$ with a $\log$ parabola function:

$$
F(E)=N_{0} E_{*}^{-\left(\alpha+\beta \log _{10} E_{*}\right)}
$$

where $E_{*}=E / 300 \mathrm{MeV}, \alpha=1.78 \pm 0.04$ and $\beta=0.030 \pm 0.009$. The measured integral flux above $200 \mathrm{MeV}$ is $F(\mathrm{E}>200 \mathrm{MeV})=(15.7 \pm 0.4) \cdot 10^{-8} \mathrm{ph} \mathrm{cm}^{-2} \mathrm{~s}^{-1}$, compatible with previous results [1].

The X-ray spectrum was fitted with a broken power law with a photon index of $\Gamma_{\mathrm{L}}=2.81 \pm$ 0.14 below the energy break $E_{\mathrm{c}}=(4.33 \pm 0.33) \mathrm{keV}$ and a photon index $\Gamma_{\mathrm{H}}=3.23 \pm 0.04$ above 


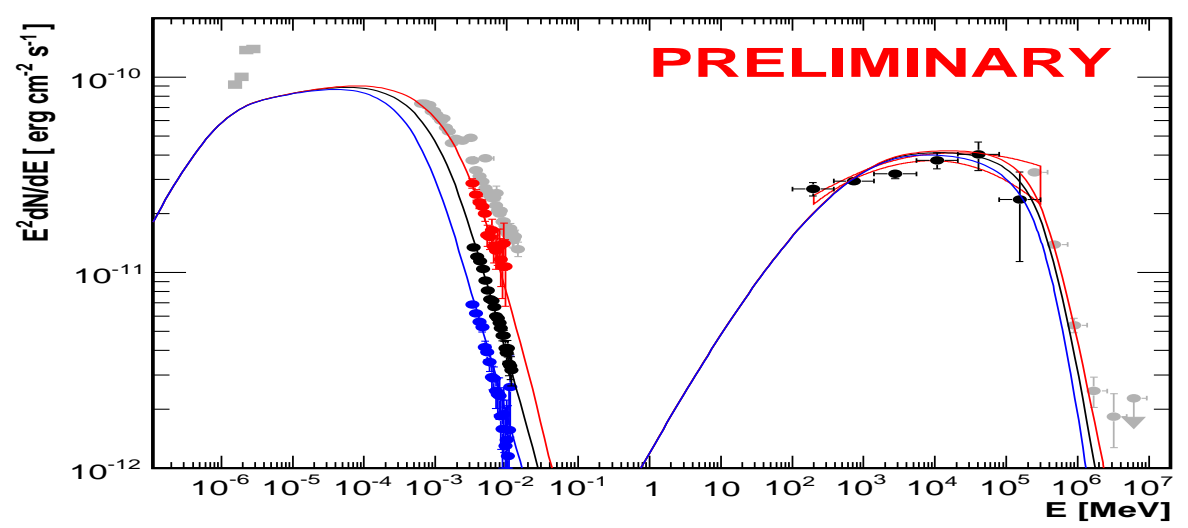

Figure 3: Spectral energy distribution (SED) of PKS 2155-304. The lines correspond to SSC calculations of the $3 \mathrm{X}$-ray different states. Black is the time-averaged X-ray flux, red corresponds to the highest flux and blue to the lowest flux.

the break while the $2-10 \mathrm{keV}$ flux is $F_{2-10 \mathrm{keV}}=(1.78 \pm 0.01) \times 10^{-11} \mathrm{erg} \mathrm{cm}^{-2} \mathrm{~s}^{-1}$ which is $\sim 3$ times lower than the $2-10 \mathrm{keV}$ flux estimated during the 2008 campaign.

The time average SED (Figure 3) was fitted with a time dependent SSC model similar to the one described in [7]. Electrons, following a distribution $Q\left(\gamma_{e}\right)$, are continuously injected into the emission region and lose energy by synchrotron and inverse Compton processes. The evolution of the overall density of electrons can be described by the Fokker-Planck equation:

$$
\frac{\partial N_{e}}{\partial t}=\frac{\partial}{\partial \gamma_{e}}\left[\left(\dot{\gamma}_{s}+\dot{\gamma}_{c}\right) N_{e}\left(\gamma_{e}, t\right)\right]+Q\left(\gamma_{e}\right)-\frac{N_{e}\left(\gamma_{e}, t\right)}{t_{e s c}}
$$

where $N_{e}\left(\gamma_{e}, t\right)$ is the density of electrons at the time $t$ and $\dot{\gamma}_{s}\left(\operatorname{Resp} \dot{\gamma}_{c}\right)$ is the energy lost by synchrotron (Resp inverse Compton) process. The system reaches the steady state when $\frac{\partial N_{e}}{\partial t}=0$.

A low $B$ field value was used $(B=0.01 \mathrm{G})$ as for the MWL campaign in 2008 [1]. The size of the region is $R=7 \cdot 10^{16} \mathrm{~cm}$ and the Doppler factor is $\delta=40$ leading to a variability time scale close to 1 day. The injection distribution $Q\left(\gamma_{e}\right)$ follows a triple power law of index $p_{0}: p_{1}: p_{2}$ between electron Lorentz factors $1: \gamma_{1}: \gamma_{2}: 10^{6.5}$. The result of the calculation is shown on Figure 3 together with calculations describing the highest and lowest X-ray fluxes measured during the MJD 54970-55040 period (section 5.1).

\section{Modeling of the variability}

\subsection{The MJD 54970-55040 Period}

The same set of parameters for the time-averaged X-ray flux was used to describe the high and low X-ray states of this period. Only the parameter $\gamma_{2}$ needs to be changed by a factor 2 from one realization to an other. A natural way to reproduce the observed behavior is to start from the high state set of parameters and vary $\gamma_{2}$ to the value used for the low state. This corresponds to a diminution of the number of high energy $\left(\gamma_{e}>\gamma_{2}\right)$ electrons. 

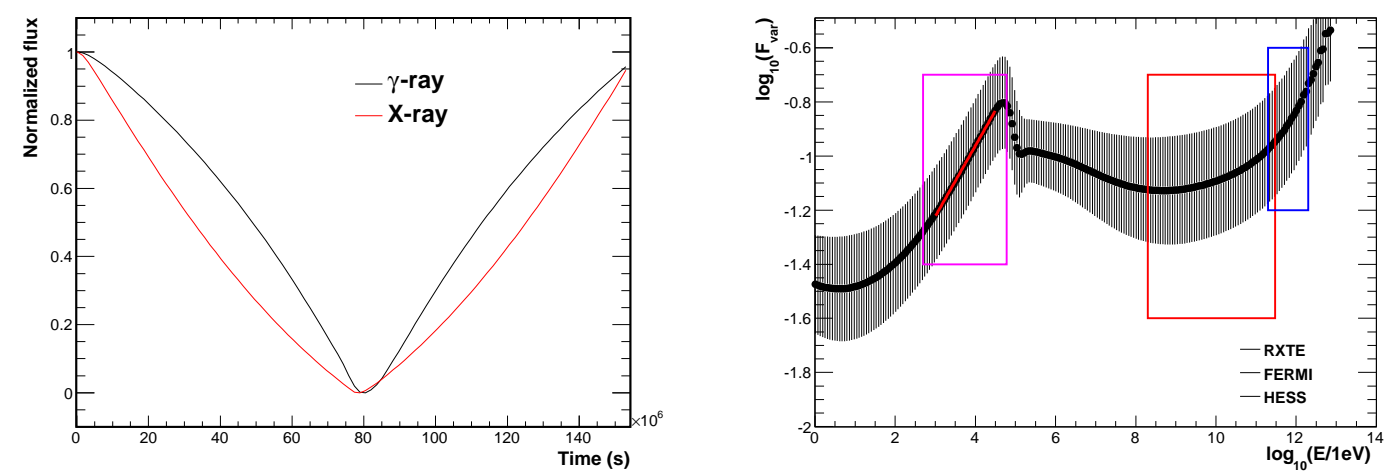

Figure 4: Left: Simulated X-ray and GeV light curves reproducing the MJD 54970-55040 Period. Right: Calculated $\mathrm{F}_{\mathrm{var}}$ as a function of the energy with our time dependent SSC model.

We found if $\gamma_{2}$ varies linearly with time, the observed profile is quantitatively reproduced contrary to a rapid change ${ }^{2}$. The resulting light curves are shown in figure 4 . The sequence is reproduced with decay faster in X-ray than in $\gamma$-ray and a faster increased of the $\gamma$-ray flux. Nevertheless more sophisticate models are required to quantitatively reproduce the data.

\subsection{Dependence of $F_{v a r}$ with energy}

The behavior of $F_{\text {var }}$ with energy can only be reproduced using a statistical approach. We used the set of parameters obtained by [5]. The injected electrons follow a relativistic Maxwellian distribution (or Pile-up distribution) [6]:

$$
Q(t, \gamma)=K \gamma^{2} \exp \left(-2 \gamma / \gamma_{b}(t)\right)
$$

which naturally arise when considering a Fermi mechanism for the acceleration with synchrotron and inverse Compton losses.

The parameter $\gamma_{b}(t)$ was chosen from a red noise distribution with an index of $\beta=-2$ and 50 light curves were simulated. For each realization, the observed flux is computed, allowing to evaluate $F_{v a r}$ as a function of the energy.

The average and the RMS of $F_{v a r}$ over the 50 simulations are shown in Figure 4. Between the optical and the X-ray bands, $\mathrm{F}_{\mathrm{var}} \propto E^{\kappa}$ with an index $\kappa=0.26 \pm 0.08$, in good agreement with the observations. In the Fermi-LAT range, $\mathrm{F}_{\text {var }}$ is less energy dependent. The general trend is also different from the measured one since our model predicts higher variability above $200 \mathrm{GeV}$ than below. This is due to the fact that most of the inverse Compton scattering take place in the Thompson regime in our model. If the scattering occurs in the Klein-Nishina regime, the variability at VHE would be reduced by the diminution of the cross section. These Klein-Nishina effects seem to have a major role in PKS 2155-304 as shown by [10].

\section{Conclusions}

We have shown the results of a monitoring campaign on PKS 2155-304 with RXTE and Fermi. Two different aspects of the variability were studied in detail: a statistical property ( $\mathrm{F}_{\mathrm{var}}$ as a func-

\footnotetext{
${ }^{2}$ faster than the variability time scale of 1 day.
} 
tion of the energy) and a specific event in the light curve (the MJD 54970-55040 Period). A time dependent SSC model has been successfully used to reproduce qualitatively the observed behavior.

In the X-ray energy range, $F_{\mathrm{var}}$ increases with the energy and follows a power law. This can be reproduce with simulation of an injection term $Q$ following a red noise. The mismatch between our model and the data at high energy might be explained by invoking Klein-Ninshina effect.

The period MJD 54970-55040 has shown a interesting sequence with a rapid decay of the $\mathrm{X}$-ray flux and a slow decrease of the $\mathrm{GeV}$ emission with a time scale of 2 weeks. This period has been reproduced by simulating a diminution of the number of high energy electrons through a linear time decrease of the $\gamma_{2}$ parameter. This implies a complex relationship between X-ray and $\mathrm{GeV}$ radiation which can only be understood with time dependent emission models.

\section{Acknowledgements}

The Fermi-LAT Collaboration acknowledges support from a number of agencies and institutes for both development and the operation of the LAT as well as scientific data analysis. These include NASA and DOE in the United States, CEA/Irfu and IN2P3/CNRS in France, ASI and INFN in Italy, MEXT, KEK, and JAXA in Japan, and the K. A. Wallenberg Foundation, the Swedish Research Council and the National Space Board in Sweden. Additional support from INAF in Italy and CNES in France for science analysis during the operations phase is also gratefully acknowledged.

\section{References}

[1] Aharonian, et al. (H.E.S.S. and Fermi-LAT Collaborations) 2009, ApJL, 696, 150, [astro-ph/0912.5152]

[2] Atwood, W. B., et al., 2009, ApJ, 697, 1071 [astro-ph/ 0902 . 1089]

[3] Arnaud, K. A. 1996, in Astronomical Data Analysis Software and Systems V, ASP Conf. Ser., 101, 17

[4] Chadwick, P. M. et al., 1999, Astroparticle Physics, 11145 [astro-ph/9812122]

[5] Giebels, B., Dubus, G., Khelifi, B., 2007, A\&A, 462, 29 [astro-ph / 0610270 ]

[6] Henri, G., Saugé, L. ApJ, 2006, 640, 185

[7] Kataoka, J. PhD thesis, University of Tokyo, 2000

[8] Li, T.-P., et al, 2004, CJAA, 4, 583

[9] Maraschi, L. et al, 2002, New Visions of the X-ray Universe in the XMM-Newton and Chandra Era, [astro-ph/0202418v1]

[10] Sanchez, D., Giebels, B., 2009, Fermi Symposium [astro-ph/0 912 . 5152]

[11] Schwartz, D. A., 1979, ApJL, 229, 53

[12] Strong, A., et al., 2004, ApJ, 613, 962 [astro-ph / 0406254 ]

[13] Vaughan S., Edelson R., Warwick R. S., Uttley P., 2003, MNRAS, 345, 1271 [astro-ph/0307420] 